\title{
Factores que inciden en el consumo de agua doméstico. Estudio a partir de un análisis bibliométrico*
}

\section{Factors that influence domestic water consumption. Study from a bibliometric analysis}

\author{
Álvaro Francisco Morote Seguido ${ }^{1}$
}

\begin{abstract}
RESUMEN
El mundo desarrollado ha vivido importantes transformaciones territoriales desde la segunda mitad del siglo xx. Algunos de estos cambios han sido el intenso proceso de urbanización, que a su vez, se ha traducido en un espectacular aumento de viviendas y población, al igual que la demanda de determinados recursos como el agua. En este sentido, la cuestión relacionada con el consumo de agua se ha convertido en un tema de estudio en las últimas décadas, principalmente, en aquellas áreas caracterizadas por la escasez de recursos hídricos y donde el proceso de urbanización ha sido más intenso. El objetivo de esta investigación es analizar y poner de manifiesto cuáles son los factores que inciden en el consumo de agua a partir de un estudio bibliométrico.
\end{abstract}

\footnotetext{
* Este artículo es resultado de la concesión de una beca pre-doctoral de Formación de Profesorado Universitario del Programa Nacional de Investigación Científica, Desarrollo e Innovación Tecnológica (FPU) y se inserta en los proyectos de investigación "Urbanización y metabolismo hídrico en el litoral de Alicante: análisis de tendencias para el periodo 2000-2010" (CSO2012-36997-CO2-02) financiado por el Ministerio de Ciencia y Tecnología y "Usos y gestión de recursos hídricos no convencionales en el litoral de las regiones de Valencia y Murcia como estrategia de adaptación a la sequía" (CSO2015-65182-C2-2-P) financiado por el Ministerio de Economía y Competitividad.

${ }^{1}$ Instituto Interuniversitario de Geografía (Universidad de Alicante). alvaro.morote@ua.es. ORCID iD: http://orcid.org/0000-0003-2438-4961.
} 
Los resultados indican una gran variedad de factores que pueden incidir en el consumo de agua y las principales áreas donde esta temática es estudiada.

Palabras Clave: agua; factores; incidencia; consumo.

\begin{abstract}
The developed world has been suffered important land uses changes since the half $\mathrm{xx}$ century. Some of these changes were the intense process of urbanization and the increase of dwellings and population and the demand of resources like water. In this sense, water consumption has been important in the studies in recent years, in general, in areas characterized by lack of water resources and where the urbanization process has been significant. The aim of this research is to analyze and highlight which factors influence water consumption from a bibliometric study. The results suggest that there are some factors that could influence water consumption and where this line of study is more important.
\end{abstract}

KEY WORDS: water; factors; incidence; consumption.

Cómo citar este artículo / Citation: Morote Seguido, Álvaro Francisco (2017): "Factores que inciden en el consumo de agua doméstico. Estudio a partir de un análisis bibliométrico", Estudios Geográficos, LXXVIII/282, pp. 257-281.

\title{
1. INTRODUCCIÓN
}

El mundo desarrollado ha vivido importantes transformaciones territoriales desde la segunda mitad del siglo xx. Algunos de estos cambios han sido el intenso proceso de urbanización, que a su vez, se ha traducido en un espectacular aumento de viviendas y población (Membrado, 2015). En este sentido, según el Programa de Desarrollo de las Naciones Unidas (UNDP), ya en el año 2000 se estimaba que el 55\% de la población mundial viviría en las ciudades en 2015. Estos cambios han sido más evidentes en países desarrollados del arco Mediterráneo como Italia (Salvati y Sabbi, 2011) y Francia, en EE.UU. (Fernández y Barrado, 2011), en Inglaterra y Australia (Hurd, 2006; Troy y Holloway, 2004). En el caso de España, estas transformaciones se han producido de manera general en las grandes conglomeraciones urbanas (Cubillo et al., 2008) y el área costera mediterránea como son las Islas Baleares (Albert y Rullán, 2007), Cataluña (Domene y Saurí, 2006), Comunidad Valenciana (Burriel, 2008; Morote, 2014), Murcia (Monreal, 2001) o Andalucía (Raya y Benítez, 2002). En definitiva, de manera general, en áreas donde la actividad turística es el principal sector económico (Rico et al., 2009). Lo que se ha pro- 
ducido es una expansión de la urbanización de baja densidad desde la década de los años sesenta (Vera, 1987), que se vincula con la presencia de espacios exteriores como jardines y piscinas, generalmente en áreas costeras ligadas con el turismo de sol y playa (Baños, 1999), que ha provocado un proceso de «litoralización» (Piqueras, 2012).

En relación con el aumento de la urbanización y la actividad turística, se ha producido un incremento del consumo de determinados recursos como es el agua (Sotelo et al., 2012). El sector turístico presenta vínculos y relaciones directas con el abastecimiento de agua, dado que éste actúa sobre los destinos en sus facetas de factor condicionante y recurso del que depende la implantación y el desarrollo de la actividad (Vera et al., 2004). Se prevé que en 2050 el consumo de agua aumentará un $44 \%$ para satisfacer las demandas industriales y de población (Fundación Aquae, 2015). A pesar de ello, existe amplia evidencia empírica sobre el descenso del consumo de agua en las últimas décadas en la mayor parte de las grandes aglomeraciones urbanas de los países desarrollados (Deoreo y Mayer, 2012; Gil et al., 2015; March et al., 2014).

Por lo tanto, una cuestión que es importante tener en cuenta en relación con el consumo de agua doméstico son los factores que puede incidir en él, y que es de vital importancia a la hora de planificar los nuevos escenarios del futuro, teniendo en cuenta una concentración de la población en las ciudades, el cambio climático, episodios de sequías, etc. En este sentido, la cuestión relacionada con los recursos hídricos se ha convertido en un tema de estudio en las últimas décadas, principalmente en territorios donde los procesos de urbanización han sido más intensos y caracterizados a su vez por la escasez hídrica.

\section{Objetivos y Metodología}

El objetivo de esta investigación es llevar a cabo una identificación de los factores que influyen en el consumo de agua doméstico a partir de un análisis bibliométrico. Para ello, se analizarán las diferentes temáticas de estudio y se evidenciará su estado actual, además de la identificación de las diferentes áreas de análisis donde se han implementado estas líneas de trabajo. Cabe, no obstante, llevar a cabo una precisión. En este sentido, las publicaciones analizadas proceden en gran medida del mundo anglosajón (EE.UU. y Australia, principalmente). Son en estos ámbitos donde los procesos de urbanización han sido más evidentes y con una mayor duración en el tiempo, especialmente, el proceso que se vincula con la urbanización de baja densidad donde las nuevas naturalezas urbanas (jardines y piscinas) se convierten en los prin- 
cipales factores consumidores de agua. Ello explica la atención que han recibido estas cuestiones; acentuadas, asimismo, por implantarse en ocasiones en territorios con estrés hídrico.

Cabe indicar la complejidad a la hora de recopilar las publicaciones y autores. Por ello, se ha realizado una aproximación a los trabajos más representativos relacionados con estas temáticas. En un primer momento se ha procedido a la búsqueda de publicaciones y autores en función de los estudios. Para la búsqueda de esta bibliografía se han utilizado buscadores de base de datos como "Scopus" y "Web of Knowledge". Entre los criterios de búsqueda se han tenido en cuenta tanto las palabras clave y autores de referencia relacionados con los factores que inciden en el consumo de agua. Las palabras claves utilizadas han sido: agua, consumo, factores, urbanización, demanda, suministro hídrico, huella hídrica, turismo, jardines y piscinas. Se ha llevado a cabo una búsqueda tanto en castellano como en inglés. De esta manera se ha podido poner de manifiesto la predominancia de publicaciones procedentes del ámbito anglosajón.

FIGURA 1

ÁREAS DE ESTUDIO IDENTIFICADAS

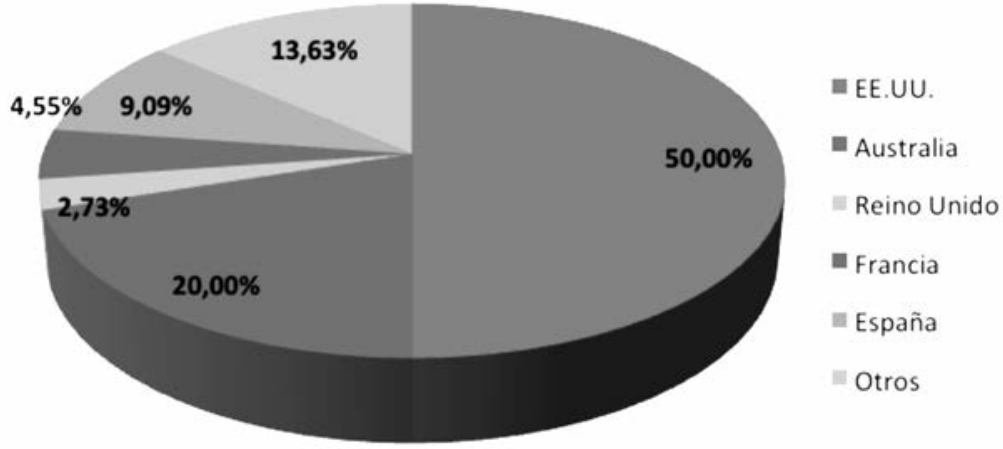

Elaboración propia a partir del análisis bibliométrico.

Una vez se ha recopilado la información necesaria (autores y publicaciones), se ha procedido a identificar grandes temáticas de estudio para poder llevar a cabo un análisis bibliográfico más exhaustivo. Las interrelaciones entre algunos aspectos considerados han determinado que, algunos autores y sus respectivos trabajos, se hayan incluido en varias áreas temáticas. También cabe hacer notar que, finalmente, se ha procedido a una preselección de aquellos 
trabajos y autores que analizan los factores que influyen en el consumo de agua doméstico desde una perspectiva geográfica y territorial y especialmente, aquéllas investigaciones que han sido de referencia para posteriores publicaciones en torno a estas líneas de estudio. Además, cabe indicar, que en este estudio no se han citado todas las publicaciones y autores preseleccionados ya que hubiera extendido el trabajo y el apartado de Bibliografía desmesuradamente.

A tenor de los resultados obtenidos en el proceso de búsqueda bibliométrica, con el objetivo de identificar las investigaciones relacionadas con los factores que pueden incidir en el consumo de agua, se han identificado las siguientes líneas temáticas: factores socio-demográficos, psicológicos, políticoeconómicos, el modelo urbano y las condiciones climáticas. Esta agrupación de trabajos se ha agrupado atendiendo a criterios que se vinculan con factores demográficos y sociales, el comportamiento de la población, la influencia de la aplicación de diferentes políticas de precios del agua, la tipología de la vivienda y el modelo urbano y finalmente, la menor o mayor influencia que puedan tener las condiciones climáticas.

El total de publicaciones preseleccionadas han sido 114 (entre artículos, capítulos de libros, congresos y tesis doctorales). Los trabajos predominantes tienen que ver con la vinculación del los factores político-económicos y el modelo urbano (el 30,70\% respectivamente). En relación con las áreas de estudio, la mayor parte de las publicaciones se han llevado a cabo en EE.UU. (50\%) y Australia (20\%), en cambio, en España sólo se vincula el 9,09\% de los trabajos (figura 1).

\section{FACTORES QUE INCIDEN EN El CONSUMO DE AGUA DOMÉSTICO}

\subsection{Consumo de agua y factores socio-demográficos}

El estudio de los factores socio-demográficos y su incidencia en el consumo de agua ha sido objeto de una atención dilatada tanto en el tiempo como en el espacio. En relación con esta interrelación, los trabajos se centran en la vinculación existente entre el número de personas por hogar, la influencia de la edad y la procedencia de los residentes (figura 2). Las áreas de estudio principales donde son tratados estos trabajos son EE.UU., destacando los Estados de Arizona (ciudades de Tucson y Phoenix) o Texas (ciudad de San Antonio), y Australia (ciudades de Adelaida y Sydney), por ser estas regiones donde más intenso ha sido el proceso de urbanización de baja densidad en las últimas décadas y por la escasez de recursos hídricos. 
FIGURA 2

PRINCIPALES LÍNEAS DE ESTUDIO QUE RELACIONAN LOS FACTORES QUE INCIDEN EN EL CONSUMO DE AGUA DOMÉSTICO

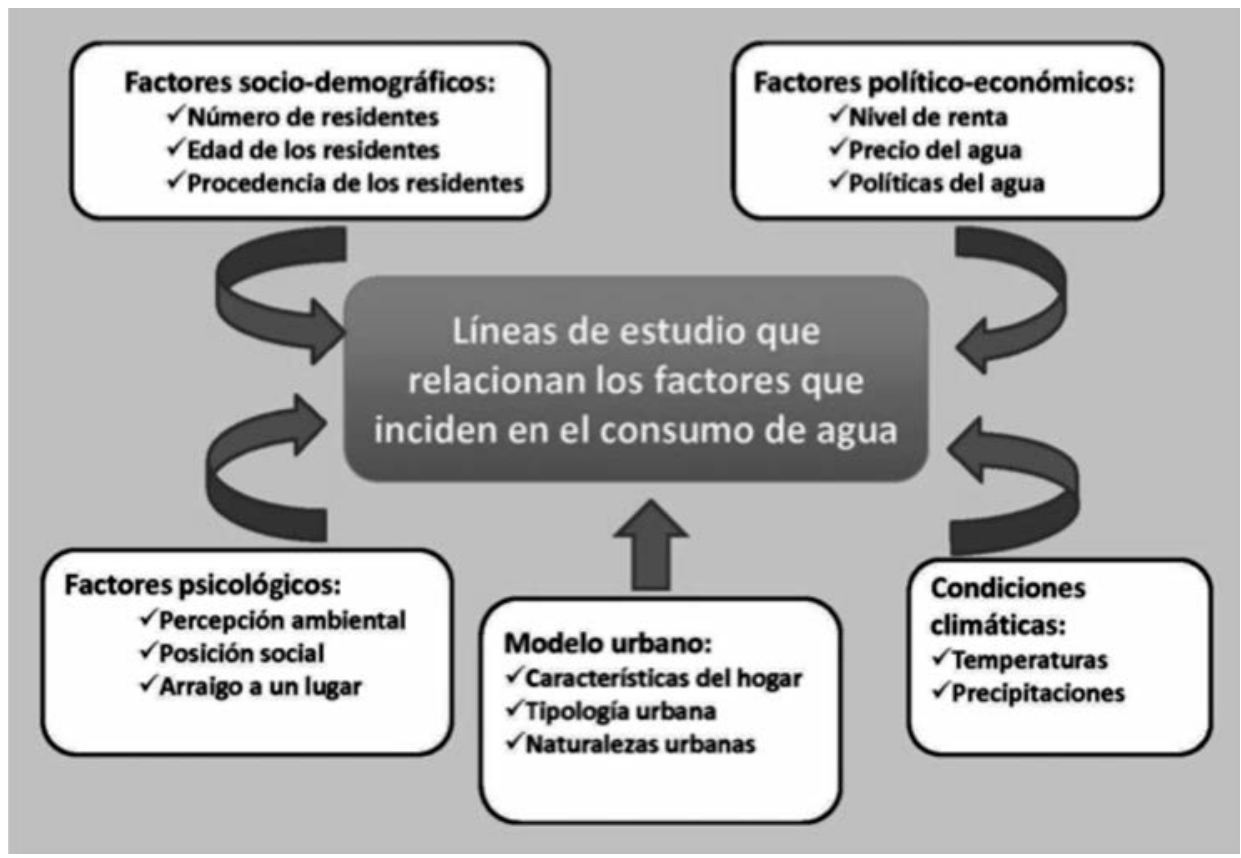

Elaboración propia a partir del análisis bibliométrico.

En relación con los trabajos que establecen un nexo entre consumo de agua y número de personas por vivienda se encuentran tesis variadas. Mitchell (2001), para el caso de EE.UU., argumenta que el descenso del número de residentes en los hogares no ha repercutido en una disminución del consumo de agua. Afirma que se ha incrementado el número de viviendas ocupadas por personas que viven individualmente. Ello ha repercutido que algunos usos del agua como puede ser el lavado de utensilios de cocina, ropa o elementos externos de la vivienda (jardín y piscina) no se compartan. Hecho, que sí que ocurre, por ejemplo, en viviendas donde residen familias con 3 o más miembros. Un ejemplo de lo comentado anteriormente es un informe elaborado en 2011 por el Ayuntamiento de Londres donde queda recogido el consumo de los hogares según el número de residentes. En este sentido, se pone de manifiesto que en los hogares donde reside un habitante, el consumo es de 207 li- 
tros/hab/día, en cambio, se reduce a 145 litros/hab/día en los hogares con 3 habitantes y 127 en hogares con 6 residentes. En cambio, otras investigaciones relacionan directamente el incremento del consumo de agua conforme aumenta el número de personas que componen el hogar (Dandy et al., 1997; Höglund, 1999; Keshavarzi et al., 2006; Nieswiadomy y Cobb, 1993). De manera general, las publicaciones se relacionan con EE.UU. y Australia, si bien, hay algunas como la de Keshavarzi et al., (2006), que tienen como área de estudio Irán, país que por su modelo social y económico, contrasta con la mayoría de los estudios. En este trabajo se obtuvieron resultados equiparables a los de ámbitos anglosajones. Así, por ejemplo, los resultados de esta investigación revelaron que el consumo de agua en las viviendas de baja densidad (chalés) era de 121,7 litros/habitante/día. Además, demostraron que en estos hogares, una de las principales variables que repercutían en el consumo de agua (además del tamaño y riego del jardín) era el número de miembros que componía la familia.

Un segundo factor socio-demográfico identificado que se vincula con el consumo de agua es la edad de los residentes. Nauges y Thomas (2000) y Troy et al., (2005), para el caso de Francia y Australia respectivamente, argumentan que en las viviendas donde habita población más joven, el consumo medio de agua es mayor que en los hogares donde residen personas mayores. Ello se debe a la mayor frecuencia de duchas y un consumo mayor de los usos exteriores del agua con fines recreativos. En el caso de España, Gil et al., (2015) manifiestan que uno de los motivos por el que se ha podido reducir el consumo de agua doméstica en el litoral mediterráneo ha sido causado por el envejecimiento de la población y la pérdida de población en las cohortes más jóvenes por la emigración debido a la crisis económica de 2007/08. En este sentido, pusieron de manifiesto que una persona de 65 años o más consume un $25 \%$ menos de agua que en el segmento anterior. Numerosas son las publicaciones que insisten en esta línea. En ellas, se afirma que las personas mayores tienen una actitud más ahorradora en el consumo de agua. Otros estudios como el de Gregory y Di Leo (2003) en Shoalhaven (Australia) y el de Gilg y Barr (2006) en Devon (sur-oeste de Inglaterra) siguen esta misma línea argumental, es decir, los residentes con mayor edad muestran unos hábitos de consumo más favorables al ahorro de agua. Otros trabajos, en cambio, han mostrado que en las viviendas con jardín donde residen personas mayores, el consumo de agua aumenta debido al riego del espacio ajardinado (Lyman, 1992). Ello se debe por la afición a la jardinería, tan arraigada en el mundo anglosajón. También se encuentran estudios con resultados diferentes a los anteriores. Mayer et al., (1999), en una investigación llevada a cabo en un 
conjunto de ciudades norteamericanas (Denver, San Diego, Tampa, etc.), argumentan que la población joven, concretamente los niños, consumen menos agua del grifo que adolescentes y adultos.

Otros estudios han centrado el consumo de agua doméstico con la procedencia de los residentes. Al igual que en la anterior variable, se encuentran disparidad de opiniones. Por un lado destacan los trabajos de Griffin y Chang (1990) y Gaudin et al., (2001) en el Estado norteamericano de Texas. Estos autores argumentan que los mayores consumos de agua se dan en aquellas viviendas donde reside la población inmigrante latina. De Oliver (1999), demostró que en la ciudad de San Antonio (Texas, EE.UU.), en aquellos lugares donde se llevaban a cabo campañas divulgativas destinadas al ahorro del agua, los niveles de ahorro que se consiguen pueden variar entre unas etnias u otras.

Otros autores como Nauges y Reynaud (2001) argumentan en su investigación realizada en el Départament de la Moselle y el Départament de la Gironde (Francia) que los inmigrantes procedentes de los países en vías de desarrollo se caracterizan por tener un comportamiento más austero en el consumo de agua. Wentz y Gober (2007) demostraron en un estudio llevado a cabo en la ciudad de Phoenix (Arizona) que la población que se asienta en un territorio, implantan el mismo tipo de jardinería de sus países de origen. Para el caso español, por ejemplo, Morote y Hernández (2016) en la costa de Alicante, han comprobado que la población extranjera procedente del norte y centro de Europa que reside en las urbanizaciones ha modificado los patrones de ajardinamiento con tal de ahorrar agua para el riego del jardín. En este sentido, los resultados de las entrevistas llevadas a cabo en estos hogares ponen de manifiesto que en un primer momento, cuando la población extranjera se compraba un chalé, instalaban un jardín de tipo atlántico donde el césped era el principal elemento de vegetación. En cambio, con el paso de los años y con el incremento del precio del agua, el coste del mantenimiento del jardín y la escasez de recursos hídricos, ha provocado la mutación de un jardín atlántico por uno mediterráneo. Ejemplo de ello son los argumentos que quedaron recogidos por los entrevistados: «no riego el jardín porque tengo plantas crasas», «en España hace mucho calor para tener plantas de tipo atlántico»o «en el jardín tengo árboles y arbustos que sólo se riegan con el agua de la lluvia». También cabe indicar el trabajo de Smith y Ali (2006) llevado a cabo en distintas ciudades de Reino Unido donde analizaron la influencia étnica y religiosa en el consumo de agua. Interesantes resultan, entre otros, los datos que vinculan el descenso del consumo de agua en los barrios donde predominan residentes musulmanes coincidiendo con la celebración del Ramadán. 


\subsection{Consumo de agua y factores psicológicos}

En relación con el consumo de agua y los aspectos psicológicos, las áreas de estudio principales identificadas se vinculan con EE.UU. (Estados de Arizona y Nuevo México) y Australia (ciudades de Sydney, Melbourne y Perth). Destacan varias líneas de trabajo, a saber: estudios que tratan de vincular el consumo de agua desde la psicología ambiental (percepción y concienciación ambiental), la posición social y el arraigo de un lugar. Entre las investigaciones que relacionan el nexo consumo de agua y percepción ambiental, destaca la de Jorgensen et al., (2009). Estos autores analizaron los diferentes factores que se relacionan con la percepción que tienen los residentes en función de los comportamientos de sus vecinos en torno al uso de agua. Argumentan que se puede producir un aumento del consumo si el resto de los vecinos tiene un mal uso del agua, ya que percibir que otros no ahorran agua disminuye la motivación de la buena gestión de este recurso, y por lo tanto, puede aumentar dicho consumo. En esta misma línea, la investigación de Aitken et al., (1991), llevada a cabo en la ciudad de Melbourne (Australia), comprobaron que los residentes de los hogares tienden a consumir agua al mismo nivel que sus vecinos. Askew y McGuirk (2004) analizaron también en Australia, que los cambios en las actitudes o los comportamientos por cuenta propia del individuo con el fin de adaptarse a unas determinadas normas sociales, puede incrementar los usos domésticos de agua, ya que éstas pueden influir la frecuencia y los patrones de uso del recurso, especialmente en los espacios ajardinados.

En relación con la concienciación ambiental, son numerosos los estudios que se han llevado a cabo (Gardener y Stern, 1996; Kiesling y Manning, 2010; Larson et al., 2009; Yabiku et al., 2008), al igual que las diferentes medidas adoptadas para ahorrar agua (Randolph y Troy, 2008; Savenije y Van der Zaag, 2002). Randolph y Troy (2008) ponen de manifiesto que el suministro de agua en la ciudad de Sydney se encuentra bajo una gran presión ya que la demanda de agua sigue en aumento. Estos autores argumentan que las estrategias de reducción de la demanda han tenido cierto éxito, pero el consumo interno sigue siendo alto. En este trabajo se analizaron las actitudes de los residentes de los hogares en relación con el consumo de agua y evidenciaron que la demanda de agua estaba fuertemente vinculada con los diferentes tipos de vivienda, aspectos culturales, institucionales, etc.

La segunda línea temática identificada tiene que ver con la posición social (prestigio) de los residentes, es decir, un comportamiento que se relaciona con un mayor consumo de agua debido a la presencia de elementos consumidores de este recurso (piscinas, jacuzzis, jardines con vegetación exuberante, elec- 
trodomésticos, etc.). Este factor también cabe mencionar que está fuertemente interrelacionado con el factor económico (ver Sotelo et al., 2010). Numerosas son las investigaciones que se dedican al análisis de estos factores (Bhatti y Andrew, 2004; Larsen y Harlan, 2006; Syme et al., 2004). Estos últimos, investigaron específicamente el uso de agua en el exterior de la vivienda para el caso de la ciudad de Perth (Australia), llegando a la conclusión, de que el estilo de vida (ocio y recreación del jardín) repercute en el incremento del consumo de agua, principalmente para los elementos exteriores de la vivienda (jardín y piscina).

El jardín, como elemento externo, permite evidenciar fácilmente el estatus social, entendido en términos de nivel de ingresos de sus propietarios. En esta línea, también destacan los trabajos que argumentan que el ahorro de agua se produce gracias a la instalación de medidas o dispositivos ahorradores; vinculando su instalación con la capacidad adquisitiva de los propietarios. Entre estas investigaciones se encuentran, por ejemplo, la de Geller et al., (1983) llevada a cabo en EE.UU. Estos autores argumentan que la instalación de estos dispositivos provoca un aumento en el consumo de agua, al contrario de lo que se podía esperar. Es decir, los usuarios son conscientes de que están ahorrando agua, pero esta percepción hace que estén más tiempo y con una mayor frecuencia consumiendo agua, fenómeno conocido como indica García Acosta (2012) offseting behaviour (comportamiento de compensación).

Un último factor que ha sido considerado al analizar los aspectos psicológicos, y que condiciona el consumo de agua es el arraigo del lugar. Estudios como los de Proshanky et al. (1983) y Bonaiuto et al. (2008), llevados a cabo en EE.UU. e Italia, respectivamente, argumentan que los residentes que sienten un arraigo especial en el lugar donde viven tienen unos comportamientos pro-ambientales, es decir, tienen una mayor concienciación ambiental, incluso la vegetación que hay presente en sus jardines suele ser la autóctona, y por lo tanto, la más adaptada al medio y menos consumidora de agua. Este hecho se puede constatar en el estudio de Morote y Hernández (2014) donde demostraron el cambio de la tipología del jardín de los propietarios extranjeros del centro y norte de Europa a favor de una vegetación mediterránea y adaptada al clima del litoral de Alicante.

\subsection{Consumo de agua y factores político-económicos}

La tercera línea identificada tiene que ver con la relación existente entre consumo de agua y factores político-económicos. Las investigaciones se vin- 
culan en torno al nivel de renta de las familias, el precio del agua y las políticas económicas. Líneas de trabajo de dilatada trayectoria en las investigaciones en el ámbito anglosajón, especialmente en EE.UU., donde destacan los Estados de Arizona y Colorado por el número de contribuciones.

En relación con el nivel de renta de las familias, son numerosos los trabajos que argumentan y justifican que conforme aumentan los ingresos económicos también lo hace el consumo de agua (Agthe y Billings, 2002; Chicoine y Ramamurthy, 1986; Dalhuisen et al., 2003; Dandy et al., 1997; García y Reynaud, 2003; Harlan et al., 2009; Höglund, 1999; Nieswiadomy y Cobb, 1993; Worthington y Hoffman, 2008). Cabe insistir la relación de renta económica con la presencia de elementos externos de la vivienda que consumen agua (jardines y piscinas). Hay trabajos que justifican que la presencia del jardín se vincula por el aumento del valor de la vivienda, y que además, puede ser un factor que conlleve a incrementar el precio de venta del hogar (Syme et al., 1980). Otros autores han relacionado, asimismo, el incremento del consumo de agua de la vivienda con la presencia de un mayor número de electrodomésticos (lavadoras, lavavajillas, etc.), es decir, a mayor poder adquisitivo, mayor accesibilidad y número de estos aparatos que utilizan agua. También cabría matizar que actualmente, y desde hace más de una década, los electrodomésticos han mejorado considerablemente en temas de eficiencia en el uso del agua. En este sentido, en los estudios de Gil et al., (2015) y el de Domene et al., (2004), llevados a cabo en el litoral mediterráneo español, se llegó a la conclusión de que el uso de nuevos electrodomésticos, más eficientes que los antiguos, es uno de los factores responsables de la reciente disminución del consumo de agua doméstico, ya que esta nueva tecnología puede representar hasta un ahorro del $40-60 \%$ respecto a los aparatos antiguos.

En contraposición a los argumentos anteriores, hay autores que afirman que en los hogares donde se dan los mayores ingresos económicos se produce un menor consumo de agua debido a una mayor concienciación ambiental y electrodomésticos más eficientes en el uso de agua. Cabe mencionar el estudio de Flack y Greenberg (1987), quienes estudiaron este factor en el Estado de Colorado (EE.UU.). En su investigación, llevaron a cabo encuestas en siete comunidades del noreste de este Estado para determinar si existen relaciones entre la concienciación ambiental y las variables socio-económicas. Entre las alternativas de esa actitud pro-ambiental se incluían las restricciones en el uso del agua, restricciones en el tamaño de la superficie del césped en el jardín (a menor tamaño de este elemento menor consumo de agua), y la instalación de elementos y dispositivos ahorradores de agua (Dalhuisen et al., 2003; Gaudin, 2006; Maddaus, 2001; Yepes y Dianderas, 1996). En relación con estos dispo- 
sitivos también destacan Hines et al., (1987) y Berk et al., (1993) para el área de Los Ángeles y la Bahía de San Francisco (California), respectivamente.

Millock y Nauges (2010), en su estudio realizado en Francia, llegaron a la conclusión de que las actitudes ambientales y el estado de la propiedad son elementos que influyen a la hora de la adopción de equipos de bajo consumo de agua. Además, pusieron de manifiesto que en los hogares de mayor renta económica había una probabilidad mayor de invertir en equipos más eficientes en el consumo de agua en comparación con los hogares de menor renta. En relación con el ahorro de agua, De Oliver (1999), en su investigación en la ciudad de San Antonio (EE.UU.), sin embargo, encontró resultados contradictorios con los estudios anteriormente citados ya que identificó grupos de población con ingresos económicos altos, que coincidían con aquellos que más agua despilfarraban.

El factor precio del agua es otro de los aspectos que ha sido objeto de atención en numerosas publicaciones (Dalhuisen et al., 2003; Renwik y Archibald, 1998; Thomas y Syme, 1988). En el estudio de Renwik y Archibald (1998), llevado a cabo en California, los autores llegaron a la conclusión de que un incremento del precio del agua repercutía en un descenso del consumo hídrico, especialmente en los hogares con menor renta económica. Dicho consumo, al responder al nivel de renta y, con ello, a la calidad de la vivienda, dotaciones sanitarias de la misma, espacios verdes, piscina y otras posibles instalaciones acordes con el poder adquisitivo del titular de aquélla, es un indicador digno de la mayor atención para un mejor conocimiento de la diferenciación social del espacio. En este sentido, en comparación con el litoral mediterráneo español, Gil et al., (2015), analizaron que una de las posibles causas del descenso del consumo doméstico de agua ha sido la pérdida del nivel de renta, especialmente en las clases medias. Además, los autores ponen de manifiesto que esta reducción haya podido resultar más o menos afectada por crisis económica iniciada en 2007/08, así como en qué medida ésta pueda o no haber incidido en el nivel de calidad de vida, en buen número de casos nivel de subsistencia, con la adopción de estrategias o actuaciones para reducir o, en determinados supuestos, rebajar o evitar fraudulentamente el recibo de agua.

Otros estudios se vinculan con los instrumentos de la política económica para lograr un uso sostenible del agua. Dalhuisen et al., (2009), en un estudio llevado a cabo en varias ciudades europeas, ofrecen un análisis del mercado del agua para los usuarios finales, dedicando especial atención a las características relevantes de la demanda y la oferta, incluidos los fallos de mercado que justifican la intervención del gobierno para regular este mercado que satisface los criterios básicos de sostenibilidad. También Dalhuisen et al., (2002), analizaron las posibilidades de los instrumentos de fijación de precios, la privatiza- 
ción y la liberalización de contribuir a un uso sostenible del agua. Dicha investigación lleva a cabo una discusión teórica de los principios de un sistema de arancel óptimo, la capacidad de respuesta de los consumidores a los cambios de precios, conceptos como la recuperación de los costes sociales y las posibilidades y conveniencia de los cambios en la gobernanza estructural, teniendo en cuenta las características específicas del sector del agua para mejorar la sostenibilidad del uso del agua en las zonas urbanas. Para el caso de España, destaca el estudio de Sánchez y Blanco (2012) donde se argumenta que las tarifas y el precio del agua son considerados ambos como una herramienta de control del consumo.

\subsection{Consumo de agua y urbanización}

El análisis de la relación entre consumo de agua y urbanización ha sido objeto de estudio desde numerosas perspectivas y diversidad de líneas temáticas. Por un lado se encuentran trabajos que relacionan las características de las viviendas (antigüedad, número de habitaciones, número de baños, tamaño de la parcela, etc.), las tipologías urbanas y los elementos externos de las viviendas (jardines y piscinas).

En relación con las características de los hogares, en general, destacan los trabajos de Troy y Holloway (2004) y Fox et al., (2009), llevados a cabo en Australia y Europa respectivamente. En vinculación con la edad de la vivienda destacan Nieswiadomy y Molina (1989) en EE.UU. y Nauges y Thomas (2000) en Francia; según el número de habitaciones Barkatullah (1996) y Dandy et al., (1997), ambos en Australia; y según el número de baños Chicoine y Ramamurthy (1986) y Barkatullah, (1996), éste último en Australia. En síntesis, estos estudios ponen de manifiesto que a mayor número de habitaciones, cuartos de baño y viviendas más antiguas, mayor es el consumo de agua. Para el caso de los usos internos destaca la publicación de Emrath (2000) y la de Deoreo y Mayer (2012). Esta última analiza el consumo de agua para los usos internos en las viviendas unifamiliares de EE.UU. Los autores llegaron a la conclusión de que el consumo doméstico ha descendido desde 1995 y que se prevé que siga esta tendencia conforme las nuevas tecnologías para el ahorro de agua entren en el mercado. También ponen de manifiesto que un elemento a tener en cuenta es la recesión económica que comenzó en 2007/08 y que está contribuyendo a ese descenso del consumo. También son numerosos los estudios que argumentan que se produce un mayor consumo de agua conforme aumenta el tamaño de la parcela de la vivienda (Dandy et al., 1997; Plint, 1999; Troy y Ho- 
lloway, 2004), el tipo de propiedad del hogar (es decir, en propiedad o alquiler) (Clarke et al., 1997; Gardener y Stern, 1996; Nieswiadomy y Cobb, 1993) o la ubicación del hogar (Troy y Holloway, 2004). Por ejemplo, en éste último, se analizaron los patrones de consumo de agua para los diferentes tipos de viviendas en la ciudad de Adelaida (Australia). Llegaron a la conclusión de que el consumo de agua varía entre los diferentes tipos de viviendas residenciales, y que el conocimiento de estos patrones de consumo a nivel local les permitía a los planificadores y administradores fomentar iniciativas específicas destinadas a reducir el consumo de agua y a planificar políticas ambientales. En definitiva, estudios con el objetivo de identificar la relación existente entre consumo de agua y las características del hogar.

Una segunda línea identificada tiene que ver con el nexo consumo de agua y modelo urbano, es decir, la repercusión que tiene una determinada tipología urbana en el consumo hídrico. En este sentido, se encuentran investigaciones como las de Troy et al., (2005) o la de Randolph y Troy (2008), ambas llevados a cabo en Australia. Este último, realizó un estudio en Sydney para investigar la tendencia del consumo de agua doméstico. La metodología consistió en el análisis de los datos recogidos a partir de la información proporcionada por los propietarios de las viviendas en función de la tipología urbana, además, teniendo en cuenta el perfil socio-cultural, el comportamiento en torno al uso del agua y las medidas llevadas a cabo para reducir el consumo hídrico. En el caso español, por ejemplo, destacan los trabajos en el Área Metropolitana de Barcelona (Domene y Saurí, 2006; Domene et al., 2004), en Girona (García Acosta, 2013), o la provincia de Alicante como el estudio de Rico (2007) y Gil et al., (2015). Estos últimos analizaron y compararon la tendencia del consumo de agua doméstico por tipología urbana y renta económica en la ciudad de Alicante y el Área Metropolitana de Barcelona. Llegaron a la conclusión de que se ha producido un descenso del consumo de agua, tanto doméstico como no doméstico a partir del 2004, y descartando como principal causa la crisis económica iniciada en 2007/08.

Una tercera línea temática identificada se relaciona con los elementos externos de las viviendas donde los jardines y las piscinas cobran protagonismo. En este sentido, destacan trabajos relacionados con las piscinas como el de Mayer et al., (1999), llevados a cabo en EE.UU., Syme et al., (1990-91) en Australia, Wolf y Holf (2013) en las Islas Baleares, Vidal et al., (2011) en el Área Metropolitana de Barcelona o Morote et al., (2017) en Alicante. Wentz y Gober (2007), en su estudio realizado en Arizona (EE.UU.), estudiaron los factores determinantes de los consumos de agua de las viviendas de las urbanizaciones, prestando especial atención a los usos exteriores (principalmente 
las piscinas). Una mayor atención han recibido los trabajos relacionados con las características de los jardines y su incidencia en el consumo de agua doméstico (Hurd, 2006; Mustafa, et al., 2010; Yabiku et al., 2008), debido al interés justificado, en gran medida, por los significativos incrementos de volúmenes de agua consumidos por estos elementos. En el caso de España, destacan las investigaciones en la región mediterránea como son los trabajos de Domene y Saurí (2003), Domene et al., (2005), Fernández et al., (2011), García Acosta (2014), Hof y Schmitt (2011), Morote y Hernández (2014) o Padullés et al., (2014). En definitiva, investigaciones con el objetivo de aumentar en el conocimiento de estas nuevas naturalezas urbanas, consideradas como uno de los principales elementos consumidores de agua en el exterior de los hogares. Siguiendo con la relación consumo de agua y espacios ajardinados destacan principalmente los trabajos que tratan el tipo de vegetación y su incidencia en el consumo hídrico (Hope et al., 2003; Larsen y Harlan, 2006; Larson et al., 2009), y en especial los que se relacionan con el césped (Chestnutt y Mcspadden, 1991; Parés et al., 2013; Renwick y Archibald, 1998; Swyngedouw, 1999) como vegetación predominante y su asociación a los denominados jardines atlánticos.

Hurd (2006) analizó en Australia como los jardines influyen en el incremento del consumo de agua. Este autor llegó a la conclusión de que el riego del jardín supone más de $50 \%$ del total del agua consumida en un hogar, y que la tipología del espacio ajardinado estaba influenciada por el coste del agua, el nivel educativo y cultural de los residentes. También destacan aquellos estudios que tratan las fuentes de abastecimiento (Loh y Coghlan, 2003) y los sistemas de riego (Chesnutt y McSpadden, 1991; Chesnutt et al., 2008; Syme et al., 2004). Estos últimos, por ejemplo, estudiaron la gestión de los sistemas de riego (programas de detección y reparación de fugas, reciclaje del agua, etc.), además de analizar los costes y beneficios de la práctica de estos sistemas, al igual que una mejora en la evolución de estas prácticas. Algunos estudios relacionados con esta temática es la publicación de Emrath (2000). En su estudio se calculó el consumo medio por vivienda y día en distintas ciudades de EE.UU. y Canadá en el año 2000. El resultado fue de 1.548 litros/viv/día, de los cuales, 874,5 litros son destinados a usos exteriores del hogar (el 56,45\%).

\subsection{Consumo de agua y condiciones climáticas}

Una quinta y última línea temática identificada es la que relaciona los trabajos en torno al consumo de agua y las condiciones climáticas. Principal- 
mente se vinculan con la incidencia que pueden tener las temperaturas y las precipitaciones. En este sentido, de manera general, los autores que tienen como finalidad poner de manifiesto la repercusión de las temperaturas en el consumo hídrico, argumentan que a mayor temperatura, mayor es el consumo de agua, mientras que los que vinculan el efecto de las precipitaciones, conforme éstas son mayores, menor será el consumo.

Loh y Coghlan (2003), para el caso de Australia, explican que el consumo se intensifica en verano por la mayor frecuentación de duchas, lavado y uso del agua en elementos externos del hogar. Numerosos son los trabajos que investigan la repercusión de las lluvias con el consumo de agua, aunque cabe indicar que en muchos de los estudios, también se tiene en cuenta la incidencia de las temperaturas (Darrel et al., 2011; García y Reynaud, 2003; Griffin y Chang, 1990; Miauo, 1990; Moncur, 1987; Nieswiadomy, 1992; Renwick y Archibald, 1998; Timmins, 2002). Interrelación importante, especialmente, en climas semiáridos donde el consumo de agua se acentúa en el caso de los elementos externos de las viviendas (jardín y piscina) por las pérdidas asociadas a la evapotranspiración, en el primero, y la evaporación de la lámina de agua, en la segunda. Por ejemplo, Maidment y Miaou (1986) estudiaron los datos del uso de agua diario de distintas ciudades de EE.UU. (Estados de Florida, Pennsylvania y Texas). Llegaron a la conclusión de que la respuesta dinámica del uso del agua de lluvia y la temperatura del aire es similar en las ciudades del centro de cada estado, además de las respuestas de las ciudades de Texas y Florida que eran muy similares entre sí. En cambio, la respuesta de las ciudades de Pennsylvania era más sensible a la temperatura del aire y en menor medida, a la lluvia. Sí que demostraron que había un aumento del consumo de agua cuando la temperatura máxima oscilaba entre los $4-21^{\circ} \mathrm{C}$. Si esta temperatura oscilaba entre $29-32^{\circ} \mathrm{C}$, el uso del agua se incrementaba 3-5 veces más por cada grado (en Texas y Florida). Otro estudio como el de Gober (2010), puso de manifiesto que en los entornos urbanos de las regiones áridas, éstos son vulnerables a la futura escasez de agua, debido según se explica, al cambio climático, ya que éste amenaza con reducir la oferta y aumentar la demanda (AEMET, 2015; ESPON-Climate, 2013).

Cabe citar el estudio realizado por Gil et al., (2015), que para el caso de la ciudad de Alicante, con unas condiciones térmicas y pluviométricas similares a las que se pueden encontrar en zonas de Australia o EE.UU., se analiza como las condiciones climáticas son una de las variables que pueden influir en la tendencia del consumo hídrico. En este sentido, se pone de manifiesto que estas variables son unas de las responsables del descenso del consumo doméstico entre 2012 y 2013, ya que en 2013, en los meses tardo-estivales apenas llovió, 
sólo 11,40 mm de septiembre a noviembre (3,00 en septiembre, 0,10 en octubre, casi inapreciable, y 8,30 en noviembre). Por lo tanto, un otoño seco pero a pesar de ello, con temperaturas inferiores a las de 2012, lo que invita a pensar en situaciones del oeste, sin lluvias y temperaturas menores, con cielos más o menos cubiertos, menos sol y la ciudad desambientada, por lo tanto, un tiempo nada propicio para que incremente el consumo de agua.

\section{Conclusiones}

El mundo desarrollado ha sufrido notables transformaciones territoriales desde la segunda mitad del siglo xx. De manera general, se han visto afectados las conglomeraciones urbanas y los espacios turísticos de sol y playa, que además, se suelen caracterizar por la escasez de recursos hídricos. Ejemplo de ello son los EE.UU. (Estados de Arizona, Texas, California o Florida), territorios semiáridos de Australia o parte del mediterráneo europeo. En estos territorios ha sido muy evidente el crecimiento urbano y la concentración de población. Con ello, se ha vinculado un incremento de la demanda de determinados recursos como es el agua. Por ello, los recursos hídricos se han convertido en una temática de estudio de primer orden en los trabajos que se vienen realizando desde la década de 1980 y desde diferentes enfoques geográficos, sociológicos, económicos y ambientales.

Las principales líneas inidentificadas que influyen en el consumo de agua doméstico han sido cinco: factores socio-demográficos, psicológicos, políticoeconómicos, el modelo urbano y las condiciones climáticas. Aunque también cabe hacer notar que en algunos trabajos se interrelacionan estos factores, como pueden ser los económicos con los sociales, los económicos con el modelo urbano o el modelo urbano con los sociales. La mayoría de los estudios identificados se relacionan con el mundo anglosajón, especialmente en las áreas áridas y semiáridas de territorios de EE.UU. y Australia, donde los procesos urbano-turísticos han sido más evidentes y sobre todo, por las características climáticas, es decir, escasez de precipitaciones y de recursos hídricos. Por lo tanto, son áreas con estrés hídrico y según las previsiones, más vulnerables al cambio climático debido a que se prevé una mayor concentración de población en entornos urbanos donde hipotéticamente la oferta de agua descenderá debido a una mayor escasez e irregularidad de las precipitaciones, además, de que en estos territorios habrá una mayor tensión por el recurso agua para abastecer la demanda urbana y turística, y por otra, la agraria. Hecho que ya se está produciendo, por ejemplo, en el sureste español, con la re- 
ducción de transferencias hídricas (Trasvase Tajo-Segura), debido al descenso de agua embalsada en los pantanos de cabecera (Entrepeñas y Buendía) por los intensos periodos de sequía de 2014-17. Ello ha repercutido en que la temática del consumo de agua sea más evidente en estas áreas. Para el caso español, especialmente destacan las grandes áreas urbanas (Madrid y Barcelona) y el arco mediterráneo por la gran difusión de los usos urbano-turísticos y escasez de agua.

El resultado del análisis de los factores que inciden en el consumo de agua a partir de un estudio bibliométrico pone de manifiesto la evolución seguida en las últimas décadas en relación con las líneas temáticas y sobre todo, la distinta incidencia que puede afectar en mayor o menor grado la tendencia del consumo de agua. Interesantes resultan los resultados obtenidos, por ejemplo, en relación con los factores socio-demográficos (número de residentes por hogar, edad de los residentes y procedencia) donde distintos trabajos argumentan tesis variadas. Por ejemplo, hay autores que argumentan que la población joven consume más agua, en cambio, otros ponen de manifiesto que donde reside población mayor, el consumo de agua se incrementa, especialmente si residen en viviendas con jardín.

Por lo tanto, se encuentran factores estrechamente interrelacionados, en este caso, socio-demográficos y tipología urbana. Igual sucede con las variables económicas (nivel de renta), donde se pueden observar distintos argumentos y resultados según autores. En este sentido, hay trabajos que ponen de manifiesto que en las viviendas de mayor renta económica, el consumo de agua se reduce debido a que hay una población más formada (mayor concienciación ambiental) y una presencia mayor de electrodomésticos más eficientes en el uso de agua. En cambio, otros autores argumentan que en estas viviendas es donde se dan los mayores consumos de agua debido a una mayor presencia de elementos consumidores de agua (jardines con presencia de césped y vegetación exuberante), además de que una mayor renta económica puede llegar a suponer mas despilfarro del recurso agua. Por ello, el conocimiento sobre los hábitos de consumo de la población y los factores que influyen en estos hábitos es de vital importancia para planificar escenarios de futuro relacionados con la demanda de agua, especialmente ante episodios de sequía. En este sentido, las aportaciones en esta línea pueden facilitar la adopción de políticas adecuadas a las realidades socio-económicas y territoriales presentes y futuras. 


\section{Bibliografía}

AEMET (Agencia Estatal de Meteorología) (2015): Proyecciones Climáticas para el siglo XXI en España, disponible en http://www.aemet.es/es/serviciosclimaticos/cambio_climat, fecha de acceso 01/09/2015.

Agthe, D. E. y Billings, R. B. (2002): "Water price influence on apartment complex water use", Journal of Water Resources Planning and Management, 128/5, pp. 366-369.

Aitken, C., Duncan, H. y Mc Marthon, T. A. (1991): “A cross-sectional regressionanalysis of residential water demand in Melbourne, Australia", Applied Geography, 11, pp. 157-165.

Albert A. y Rullán O. (2007): "Nuevo modelo de producción residencial y territorio urbano disperso (Mallorca 1998-2006)", en 9th International Geocriticism Symposium Porto Alegre, 28 May-1 June, disponible en http://www.ub.edu/geocrit/ 9porto/artigues.htm, fecha de consulta 15/10/2015.

Askew, L. E. y Mc Guirk, P. M. (2004): "Watering the suburbs: distinction, conformity and the suburban garden", Australian Geographer, 35, pp. 17-37.

Baños, C. (1999): "Modelos turísticos locales. Análisis comparado de dos destinos de la costa blanca", Investigaciones Geográficas, 21, pp. 35-58.

Barkatullah, N. (1996): OLS and instrumental variable price elasticity estimates for water in mixed-effects model under multiple tariff structure, Sydney, Department of Economics, University of Sydney, Report, $226 \mathrm{pp}$.

Berk, R. A., Schulman, D., Mc Keever, M. y Freeman, H. E. (1993): "Measuring the impact of water conservation campaigns in California", Climatic Change, 24, pp. 233-248.

Bhatti, M. y Andrew, C. (2004): "Home, the culture of nature and meanings of gardens in late modernity", Housing Studies, 19, pp. 37-51.

Bonaiuto, M., Aiello, A., Perugini, M., Bonnes, M. y Ercolani, A. P. (1999): "Multidimensional perception of residential environmental quality and neighbourhood attachment in the urban environment", Journal of Environmental Psychology, 19, pp. 331-352.

Burriel De Orueta, E. (2008): "La década prodigiosa del urbanismo español (19972006)", Scripta Nova, XII/270/64, disponible en http://www.ub.es/geocrit/sn/sn270/sn-270-64.htm, fecha de consulta 08/09/2015.

Chestnutt, T., Fiske, G., Rothstein, E., Pekelney, D. y Beecher, J. (2008): "Water efficiency programs for integrated water management", Journal/American Water Works Association, 100/5, pp. 132-141.

Chestnutt, T. y Mc Spadden, C. (1991): A model-based evaluation of westchester water conservation program (1a ed.), San Diego, A\&N Technical Services.

Chicoine, D. y Ramamurthy, G. (1986): "Evidence on the specification of price in the study of domestic water demand", Land Economics, 62, pp. 26-32.

Clarke, G., Kashti, A., Mc Donald, A. y Williamson, P. (1997): "Estimating small area demand for water: a new methodology", CIWEM'S Water and Environmental Journal, 11, pp. 186-192. 
Cubillo, F., Moreno, T. y Ortega, S. (2008): "Microcomponentes y factores explicativos del consumo doméstico de agua en la Comunidad de Madrid", Colección de Cuadernos de I+D+I, Madrid, Canal de Isabel II.

Dalhuisen, J., De Groot, H., Rodenburg, C. A. y Nijkamp, P. (2002): "Economic aspects of sustainable water use: Evidence from a horizontal comparison of European cities", International Journal of Water, 2/1, pp. 75-94.

Dalhuisen, J., Florax, R., De Groot, H. y Nijkamp, P. (2003): "Price and income elasticities of residential water demand: a meta-analysis", Land Economics, 79, pp. 292-308.

Dalhuisen, J., Nijkamp, P. y De Groot, H. (2009): "Economic market instruments and sustainable urban water use", International Journal of Environmental Technology and Management, 11/1-3, pp. 88-101.

Dandy, G., Nguyen, T. y Davies, C. (1997): "Estimating residential water demand in the presence of free allowances", Land Economics, 73, pp. 125-139.

Darrel, G., Harlan, S. L., Stefanov, W. L. y Martin, C. A. (2011): "Ecosystem services and urban heat riskscape moderation: water, green spaces and social inequality in Phoenix, USA", Ecological Applications, 21/7, pp. 2.637-2.651.

De Oliver, M. (1999): "Attitudes and inaction: A case study of the manifest demographics or urban water conservation”, Environmental and Behavior, 31, pp. 372 394.

Deoreo, W. B. y Mayer, P. W. (2012): "Insights into declining single-family residential water demands", Journal-American Water World Association, 104/6, pp. 383-394.

Domene, E. y Saurí, D. (2003): "Modelos urbanos y consumo de agua. El riego de jardines privados en la Región Metropolitana de Barcelona", Investigaciones Geográficas, 32, pp. 5-17.

Domene, E. y Saurí, D. (2006): "Urbanization and water consumption. Influencing factors in the Metropolitan Region of Barcelona", Urban Studies, 43/9, pp. 1.6051.623.

Domene, E., Saurí, D., Molina, J., et al. (2004): Estudi del consum d'aigua als edificis de la Regió Metropolitana de Barcelona: Situació actual y possibilitats d'estalvi, disponible en http://www.fundacioabertis.org/rcs_est/estudi_complet.pdf, fecha de consulta 07/11/2015.

Domene, E., Saurí, D. y Parés, M. (2005): "Urbanization and sustainable resource use: the case of garden watering in the metropolitan region of Barcelona", Urban Geography, 268, pp. 520-535.

Emrath, P. (2000): "Residential water use", Housing Economics, 48, pp.6-10.

ESPON-Climate (2015): Climate Change and Territorial Effects on Regions and Local Economies in Europe, disponible en http://www.espon.eu/main/Menu_Projects/ Menu_AppliedResearch/climate.html, fecha de consulta 10/09/2015.

Fernández, R., Ordovás, J. y Herrera, M. A. (2011): "Domestic gardens as water-wise Landscapes: A Case Study in Southwestern Europe", HorTechnology, 21/5, pp. 616-623. 
Fernández, S. y Barrado, D. A. (2011): "El desarrollo turístico-inmobiliario de la España mediterránea e insular frente a sus referentes internacionales (Florida y la Costa Azul): un análisis comparado", Cuadernos de Turismo, 27, pp. 373-402.

Flack, J. E. y Greenberg, J. (1987): "Public attitudes toward water conservation", Journal of the American Water Works Association, 79, pp. 46-51.

Fox, C., Mc Intosh, B. S. y Jeffrey, P. (2009): "Classifying households for water demand forescasting using physical property characteristics", Land Use Policy, 26, pp. 558-568.

Fundación Aquae (2015): Ahorrar agua en el inodoro, disponible en http://www.fundacionaquae.org/wiki/ahorrar-agua-en-el-inodoro, fecha de consulta 04/05/2015.

García Acosta, X. (2012): Nous procesos d'urbanització i consum d'aigua per a usos domèstics. Una exploració de relacions a l'àmbit gironí, tesis doctoral, Universitat de Girona, 613 pp.

García Acosta, X. (2013): "Urbanització difusa i consum d'aigua per a usos domèstics. Una exploració de relacions", Documents d'Anàlisi Geogràfica, 59/2, pp. 347-362.

García Acosta, X. (2014): "Jardines privados y consumo de agua en las periferias urbanas de la comarca de la Selva (Girona)", Investigaciones Geográficas, 61, pp. 55-69.

García, S. y Reynaud, A. (2003): "Estimating the benefits of efficient water pricing in France", Journal of Resource and Energy Economics, 26, pp. 1-25.

Gardener, G. T. y Stern, P. C. (1996): Environmental problems and human behavior (1 ed.), Boston, Allyn and Bacon.

Gaudin, S. (2006): "Effect of price information on residential water demand", Applied Economics, 38, pp. 383-393.

Gaudin, S., Griffin, R. C. y Sickles, R. C. (2001): "Demand specification for municipal water management: evaluation of the Stone-Geary form", Land Economics, 77, pp. 399-422.

Geller, E. S., Erikson, J. B. y Buttram, B. A. (1983): “Attempts to promote residential water conservation with educational, behavioral and engineering strategies", Population and Environment, 6, pp. 96-112.

Gil Olcina, A., Hernández Hernández, M., Morote Seguido, A. F., Rico Amorós, A. M., Saurí Pujol, D. y March Corbella, H. (2015): Tendencias del consumo de agua potable en la ciudad de Alicante y Área Metropolitana de Barcelona 2007-2013, Hidraqua, Gestión Integral de Aguas de Levante S.A. y la Universidad de Alicante, 164 pp.

Gilg, A. y Barr, S. (2006): "Behavioral attitudes towards water saving? Evidence from a study of environmental actions", Ecological Economics, 57, pp. 400-414.

Gober, P. (2010): "Desert urbanization and the challenges of water sustainability", Curr.Opin. Environment Sustainability, 2, pp. 144-150.

Gregory, G. D. y Di Leo, M. (2003): "Repeated behavior and environmental psychology: the role of personal involvement and habit formation in explaining water consumption", Journal of Applied Social Psychology, 33, pp. 1.261-1.296.

Griffing, R. C. y Chang, C. (1990): "Pretest analysis of water demand in thirty communities", Water Resources Research, 26, pp. 2.251-2.255. 
Harlan, S. L., Yabiku, S. T., Larsen, L. y Brazel, A. J. (2009): "Household water consumption in an arid city: Affluence, affordance and attitudes", Society and Natural Resources, 22/8, pp. 691-709.

Hines, J. M., Hungerford, H. R. y Tomera, A. N. (1987): "Analysis and synthesis of research on responsible environmental behavior: a meta-analysis", Journal of Environmental Education, 18, pp. 1-18.

Hof, A. y Schmitt, T. (2011): "Urban and tourist land use patterns and water consumption: evidence from Mallorca, Balearic islands", Land Use Policy, 28/4, pp. 792-804.

Höglund, L. (1999): "Household demand for water in Sweden with implications of a potential tax on water use", Water Resources Research, 35, pp. 3.853-3.863.

Hope, D., Gries, C., Zhu, W., Fagan, W., Redman, C. L., Grimm, N. B., et al. (2003): "Socieconomics drive urban plant diversity", Proceedings of the National Academy of Science, 100, pp. 8.788-8.792.

Hurd, B. H. (2006): "Water conservation and residential landscape: household preferences, household choices", Journal of Agricultural and Resource Economics, 31, pp. 21-32.

Jorgensen, B., Graymore, M. y O'toole, K. (2009): "Household water use behavior: An integrated model", Journal of Environmental Management, 91, pp. 227-236.

Kesharvarzi, A. R., Sharifzadeh, M., Kamgar, A. A., Amin, S., Keshtkar, S. y Bamdad, A. (2006): "Rural domestic water consumption behavior: A case study in Ramjerd area, Fars province, I.R. Iran", Water Research, 40, pp. 1173-1178.

Kiesling, F. M. y Maning, C. M. (2010): "How green is your thumb? Environmental gardening identity and ecological gardening practices", Journal of Environmental Psychology, 30, pp. 315-327.

Larsen, L. y Harlan, S. L. (2006): "Desert dreamscapes. Residential landscapes preference and behavior", Landscape and Urban Planning, 78, pp. 85-100.

Larson, K. L. Casagrande, D., Harlan, S. L. y Yabiku, S. T. (2009): "Residents yard choices and rationales in a desert city: Social priorities, ecological impacts, and decision tradeoffs", Environmental Management, 44, pp. 921-937.

Loh, M. y Coghlan, P. (2003): Domestic water use study: Perth, Western Australia 19982001, Perth, Water Corporation, 33 pp.

Lyman, R. A. (1992): "Peak and off-peak residential water demand", Water Resources Research, 28, pp. 2.159-2.167.

Maddaus, L. A. (2001): Effects of metering on residential water demand, tesina de master, University of California.

Maidment, D. y Miaou, S. (1986): "Daily water use in nine cities", Water Resources Research, 22/6, pp. 845-851.

March, H., Saurí, D. y Rico, A. M. (2014): "The end of scarcity? Water desalination as the new cornucopia for Mediterranean Spain”, Journal of Hydrology, 519, pp. 2642-2652.

Mayer, P. W., Deoreo, W. B., Optiz, E., Kiefer, J., Dziegielewski, B. y Davis, W. (1999): Residential end uses of water, Denver, American Water Works Association Research Foundation. 
Membrado, J. C., 2015: "Migración residencial y urbanismo expansivo en el mediterráneo Español", Cuadernos de Turismo, 35, pp. 259-285.

Miaou, S. P. (1990): "A class of time-series urban water demand models nonlinear climatic effects", Water Resources Research, 26, pp.169-178.

Millock, K. y Nauges, C. (2010): "Household adoption of water-efficient equipment: The role of socio-economic factors, environmental attitudes and policy", Environmental and Resource Economics, 46/4, pp. 539-565.

Mitchell, J. (2001): "Urban sprawl”, National Geographic, 200, pp. 48-56.

Moncur, J. (1987): "Urban water pricing and drought management", Water Resources Research, 23, pp. 393-398.

Monreal, J. (2001): Un nuevo mercado turístico: jubilados europeos en la región de Murcia, Murcia, Universidad de Murcia.

Morote, A. F., Saurí, D. and Hernández, M. (2017): "Residential Tourism, Swimming Pools and Water Demand in the Western Mediterranean", Professional Geographer, 69/1, pp. 1-11.

Morote Seguido, A. F. (2014): "Tipologías urbano-residenciales del litoral de Alicante: repercusiones territoriales", Ciudad y Territorio. Estudios Territoriales, 181, pp. 431-443.

Morote Seguido, A. F. y Hernández Hernández, M. (2014): "Jardines y urbanizaciones, nuevas naturalezas urbanas en el litoral de la provincia de Alicante", Documents d'Anàlisi Geogràfica, 60/3, pp. 483-504.

Morote Seguido, A. F. y Hernández Hernández, M. (2016): “Jardines y patrones de ajardinamiento en las urbanizaciones del litoral de Alicante", Boletín de la Asociación de Geógrafos Españoles, 70, pp. 31-56.

Mustafa, D., Smucker, T. A., Ginn, F., Johns, R. y Connely, S. (2010): "Xeriscape people and the cultural politics of turfgrass transformation", Environment and Planning $D, 28$, pp. 600-617.

Nauges, C. y Reynaud, A. (2001): "Estimation de la demande domestique d'eau potable en France", Revue économique, 52, pp. 167-185.

Nauges, C. y Thomas, A. (2000): "Privately-operated water utilities, municipal price negotiation and estimation of residential water demand: The case of France", Land Economics, 76, pp. 68-85.

Nieswiadomy, M. (1992): "Estimating urban residential water demand: effects or price structure, conservation and education", Water Resources Research, 28, pp. 609-615.

Nieswiadomy, M. y Cobb, S. (1993): "Impact of pricing structure selectivity on urban water demand", Contemporary Policy Issues, 11, pp. 101-113.

Nieswiadomy, M. y Molina, D. (1989): "Comparing residential water demand estimates under decreasing and increasing block rates using household data", Land Economics, 65, pp. 280-289.

Padullés Cubino, J., Vila Subirós, J. y Barriocanal Lozano, C. (2014): "Examining floristic boundaries between garden types at the scale", Investigaciones Geográficas, 61, pp. 71-86. 
Parés, M., March, H. y Saurí, D. (2013): "Atlantic gardens in Mediterranean climates: Understanding the production of suburban natures", International Journal of Urban and Regional Research, 37/1, pp. 328-347.

Piqueras, J. (2012): Geografía del territorio valenciano. Naturaleza, economía y paisaje, Valencia, Universidad de Valencia, 256 pp.

Plint, E. A. (1999): "Household responses to increased water during the California drought", Land Economics, 71, pp. 92-173.

Proshansky, H. M., Fabian, A. K. y Kaminoff, R. (1983): "Place identity: Physical world socialization of the self", Journal of Environment Psychology, 3, pp. 57-83.

Randolph, B. y Troy, P. (2008): "Attitudes to conservation and water consumption", Environmental Science and Policy, 11/5, pp. 441-455.

Raya Mellado, P. y Benítez Rochell, J. J. (2002): “Concepto y estimación del turismo residencial: aplicación en Andalucía", Papers de Turisme, 31/32, pp. 67-89.

Renwick, M. y Archibald, S. (1998): "Demand side management policies for residential water use: who bears the conservation burden?", Land Economics, 74, pp. 343-359.

Rico, A. M., Olcina, J. y Saurí, D. (2009): "Tourist land use patterns and water demand: Evidence from the Western Mediterranean", Land Use Policy, 26, pp. 493-501.

Rico Amorós, A. M. (2007): "Tipologías de consumo de agua en abastecimientos urbano-turísticos de la Comunidad Valenciana", Investigaciones Geográficas, 42, pp. 5-34.

Sánchez García, V. E. y Blanco Jiménez, F. J. (2012): "El uso sostenible del agua en núcleos urbanos: las tarifas como herramienta de control del consumo", Observatorio ambiental, 15, pp. 35-59.

Salvati, L. y Sabbi, A. (2011): "Exploring long term land cover changes in an urban region of Southern Europe", International Journal of Sustainable Development and World Ecology, 18/4, pp. 273-282.

Savenije, H. y Van Der Zaag, P. (2002): "Water as an economic good and demand management, paradigms with pitfalls", Water International, 27, pp. 98-104.

Smith, A. y Ali, M. (2006): "Understanding the impact of cultural and religious water use", Water and Environment Journal, 20, pp. 203-209.

Sotelo, J. A., Olcina, J., García, F. y Sotelo, M. (2012): "Huella hídrica de España y su diversidad territorial", Estudios Geográficos, 272, pp. 239-272.

Sotelo, J. A., Olcina, J., Tolón, A., Bolívar, X., García, F. y Sotelo, M. (2010): La huella hídrica española en el contexto del cambio ambiental, Madrid, Fundación MAPFRE, $216 \mathrm{pp}$.

Swyngedouw, E. (1999): "Modernity and hibridity: Nature, regeneracionism, and the production of the Spanish waterscape, 1890-1930". Annals of the Association of American Geographers, 89/3, pp. 443-465.

Syme, G. J., Kantola, S. J. y Thomas, J. F. (1980): "Water resources and the quarter acre block", en R. Thorne y S. Arden, (eds.), People and the Man Made Environment, $1^{a}$ ed., Sydney, University of Sydney, pp. 192-201. 
Syme, G. J., Seligman, C. y Thomas, J. F. (1990-91): "Predicting water consumption from homeowners attitudes", Journal of Environmental Systems, 20, pp. 157-168.

Syme, G. J., Shao, Q., Po, M. y Campbell, E. (2004): "Predicting and understanding home garden water use", Landscape and Urban Planning, 68, pp.121-128.

Thomas, F. y Syme, G. (1988): "Estimating residential price elasticity of demand for water; a contingent valuation approach", Water Resources Research, 24, pp. 1.8471.857 .

Timmins, C. (2002): "Measuring the dynamic efficiency costs of regulators preferences: municipal water utilities in the arid West", Econometrica, 70, pp. 603-629.

Troy, P. y Holloway, D. (2004): "The use of residential water consumption as an urban planning tool: a pilot study in Adelaide", Journal of Environmental Planning and Management, 47, pp. 97-114.

Troy, P., Holloway, D. y Randolph, A. B. (2005): Water use and the built environment: Patterns of water consumption in Sydney, City Futures Research, Report $n^{\circ} 1$, Kensigton, City Futures Research Centre, Faculty of Built Environment, UNSW.

Vera, J. F., Casado, J. Ma . y Ramón, A. B. (2004): "Consideraciones sobre el impacto del Plan Hidrológico Nacional en el sector turístico de la provincia de Alicante", en Repercusiones socioeconómicas del Plan Hidrológico Nacional en la provincia de Alicante, Alicante, Fundación COEPA, pp. 205-267.

Vera Rebollo, J. F. (1987): Turismo y Urbanización en el litoral alicantino, Alicante, Instituto de Estudios Juan Gil-Albert, 441 pp.

Vidal, M., Domene, E. y Saurí, D. (2011): "Changing geographies of water-related consumption: residential swimming pools in suburban Barcelona", Area, 43/1, pp. 67-75.

Wentz, E. y Gober, P. (2007): "Determinants of small-area water consumption for the city of Phoenix, Arizona", Water Resources Management, 21, pp. 1.849-1.863.

Wolf, N. y Hof, A. (2013): "Integration of the pixel and object domain for the mapping of new urban landscapes in the Mediterranean with a focus on outdoor water consumption", EARSeL eProceedings, 12/1, pp. 67-81.

Worthington, A. C. y Hoffman, M. (2008): "An empirical survey of residential water demand modeling", Journal of Economic Surveys, 5, pp. 842-871.

Yabiku, S. T., Casagrande, D. G. y Farley-Metzger, E. (2008): "Preferences for landscape choice in a Southwestern desert city", Environment and Behavior, 40, pp. 382-400.

Yepes, G. y Dianderas, A. (1996): Water and wastewater utilities indicators, 2a ed., Washington, World Bank, Water and Sanitation Division, 57 pp.

Fecha de recepción: 12 de junio de 2015.

Fecha de aceptación: 6 de octubre de 2015. 